\title{
A chelicerate-specific burst of nonclassical Dscam diversity
}

\author{
Guozheng $\mathrm{Cao}^{1 \dagger}{ }^{\dagger}$, Yang Shi ${ }^{1 \dagger}$, Jian Zhang ${ }^{1}$, Hongru Ma', Shouqing Hou', Haiyang Dong ${ }^{1}$, Weiling Hong ${ }^{1}$, \\ Shuo Chen', Hao Li', Yandan Wu', Pengjuan Guo', Xu Shao', Bingbing Xu', Feng Shi', Yijun Meng² \\ and Yongfeng Jin $^{1 *}$
}

\begin{abstract}
Background: The immunoglobulin (Ig) superfamily receptor Down syndrome cell adhesion molecule (Dscam) gene can generate tens of thousands of isoforms via alternative splicing, which is essential for both nervous and immune systems in insects. However, further information is required to develop a comprehensive view of Dscam diversification across the broad spectrum of Chelicerata clades, a basal branch of arthropods and the second largest group of terrestrial animals.

Results: In this study, a genome-wide comprehensive analysis of Dscam genes across Chelicerata species revealed a burst of nonclassical Dscams, categorised into four types—mDscam, sDscama, sDscam $\beta$, and sDscamy — based on their size and structure. Although the mDscam gene class includes the highest number of Dscam genes, the sDscam genes utilise alternative promoters to expand protein diversity. Furthermore, we indicated that the $5^{\prime}$ cassette duplicate is inversely correlated with the sDscam gene duplicate. We showed differential and sDscam- biased expression of nonclassical Dscam isoforms. Thus, the Dscam isoform repertoire across Chelicerata is entirely dominated by the number and expression levels of nonclassical Dscams. Taken together, these data show that Chelicerata evolved a large conserved and lineage-specific repertoire of nonclassical Dscams.

Conclusions: This study showed that arthropods have a large diversified Chelicerata-specific repertoire of nonclassical Dscam isoforms, which are structurally and mechanistically distinct from those of insects. These findings provide a global framework for the evolution of Dscam diversity in arthropods and offer mechanistic insights into the diversification of the clade-specific lg superfamily repertoire.
\end{abstract}

Keywords: Dscam, Gene duplication, Exon duplication, Alternative splicing, Alternative promoter, Chelicerate

\section{Background}

Alternative splicing plays an important role in the generation of proteomic diversity and genomic evolution in metazoans $[1,2]$. For example, the Down syndrome cell adhesion molecule (Dscam) gene in Drosophila melanogaster has the potential to generate 38,016 distinct mRNA and protein isoforms via mutually exclusive alternative splicing [3]. In this Dscam gene structure, 95 alternatively spliced exons are organised into exon 4, 6, 9, and 17 clusters that contain 12, 48, 33, and 2 copies, respectively. Forward genetic screening and biochemical approaches have shown that

\footnotetext{
* Correspondence: jinyf@zju.edu.cn

${ }^{\dagger}$ Equal contributors

'Zhejiang University, Institute of Biochemistry, College of Life Sciences, Hangzhou, Zhejiang ZJ310058, People's Republic of China

Full list of author information is available at the end of the article
}

this extensive diversity of Dscam encoded by a single locus is required for both nervous and immune systems. Dscam isoform diversity plays an important role in neuronal wiring and self-recognition, and the extensive diversity of Dscam isoforms has been shown to confer specificity for antigen recognition [4-13].

Vertebrate Dscams, which lack the striking diversity of their insect counterparts, are involved mainly in the developmental processes of the nervous system [14]. Interestingly, cadherin superfamily members, protocadherins (Pcdhs), might serve an analogous function in vertebrates [15-17]. Human Pcdh genes are arranged tandemly in three groups called $P c d h \alpha, P c d h \beta$, and $P c d h \gamma$, with 14, 22, and 22 repeats in their respective $5^{\prime}$ variable regions [18]. Unlike Drosophila Dscam1, these Pcdhs utilise alternative 
promoters to generate isoform diversity $[19,20]$. For both Dscams and Pcdhs, isoform expression appears largely stochastic and combinatorial rather than determinative, endowing a unique cell-surface identity for each neuron $[15,16,21,22]$. Both molecules exhibit isoform-specific homophilic binding, and they may have similar roles in the nervous system. Interestingly, Drosophila lacks the counterparts of vertebrate Pcdhs. Thus, these two phyla appear to have independently evolved similar molecular strategies for comparable roles by recruiting various molecules from different protein families [15].

As Pcdh isoform diversity is restricted to vertebrates, Dscam isoform diversity has been considered unique to arthropods [23, 24]. However, compared with the well-known phylogenetic distribution of vertebrate Pcdhs, Dscam diversification has not been studied in detail in arthropods, particularly in Chelicerata members, which represent a basal branch of arthropods and form the second largest group of terrestrial animals. It is generally recognised that Dscam protein structure is conserved across bilaterians, containing 10 immunoglobulin (Ig) domains and six fibronectin III (FNIII) repeats, with the tenth Ig domain located between FNIII 4 and FNIII 5 [14, 15, 17]. Recently, structural variants of Dscam have been found in Ixodes scapularis [24]. We identified shortened Dscam genes with tandemly arrayed $5^{\prime}$ cassettes in several Chelicerata species, similar to vertebrate clustered Pcdhs [25]. These results suggest that the structural and expansion pathways of Dscam might be diversified in Chelicerata species. However, no comprehensive view exists of Dscam diversification across the broad spectrum of Chelicerata. The rapidly increasing availability of genomic information regarding Chelicerata, particularly key clades such as the Xiphosuran horseshoe crab, will increase our understanding of Dscam diversification.

In this study, we performed a genome-wide comprehensive analysis of Dscam genes in chelicerates, which diverged from other Arthropod lineages 500 million years ago. The identified Dscam genes could be grouped into one classical (LDscam) and four nonclassical (mDscam, sDscama, sDscam $\beta$, and sDscamy) types based on their size and structure. Although the mDscam gene class includes the highest number of Dscam genes, the sDscam genes utilise alternative promoters to expand protein diversity. Collectively, these results demonstrate that Chelicerata specifically evolved different organisation and mechanisms that generated a diverse lineage-specific repertoire of Dscam isoforms. These findings highlight the rich Chelicerata-specific diversification of Dscam genes, and provide a global framework for the evolution of Dscam diversity in arthropods and bilaterians.

\section{Results}

\section{Genome-wide identification of Dscam genes across} Chelicerata species

To generate a global blueprint for Dscam diversity in Chelicerata, we performed a genome-wide analysis of Dscam homologues in representative species from each of the major clades. We examined one species of the order Xiphosura (Limulus polyphemus) and five species representing five major clades of the class Arachnida, including two Araneae (Stegodyphus mimosarum and Parasteatoda tepidariorum), one Scorpiones (Mesobuthus martensii), one Mesostigmatan (Metaseiulus occidentalis), and one Ixodidan (Ixodes scapularis) (Additional file 1: Table S1). These organisms constitute some of the major taxonomic groups of the Chelicerata subphylum that last shared a common ancestor $\sim 500$ million years ago [26], with longterm resolution (comparing the gene organisation among the five Arachnida clades and Merostomata). Using crossspecies comparisons with RNA-sequencing (RNA-seq) analyses, we identified 198 Dscam genes in six representatives of the Chelicerata species, 161 of which were novel or corrected (Fig. 1b; Additional file 2: Table S2). Our results indicated that all extant chelicerates display marked expansion of the Dscam gene family.

Phylogenetic analysis indicated that all of the Dscam proteins could be clustered into three groups: canonical Dscams and two groups of shortened nonclassical proteins (Fig. 2). The three groups differ in size, and are hereafter referred to as LDscam, mDscam, and sDscam, respectively (Fig. 1a). The sDscams lack the canonical Ig1-6,10 and FNIII 3-4,6 domains present in classical Dscam. The genes encoding these sDscams can be subdivided into three types-sDscam $\alpha, s D \operatorname{scam} \beta$, and $s D s c a m \gamma$-based on differing structures in the $5^{\prime}$ regions (Fig. 1a). sDscam $\alpha$ and $s D s c a m \beta$ are characterised by clustered cassette repeats in the $5^{\prime}$ regions, encoding one and two Ig domains, respectively. sDscamy shares similar domains with $s D \operatorname{scam\alpha }$ and $s D \operatorname{scam} \beta$, albeit without tandemly arrayed cassettes in the $5^{\prime}$ regions. Another type of shortened Dscam lacks the FNIII and Ig domains present in the C-terminal region of classical Dscam. As the sizes of these Dscams fall between those of canonical Dscam (LDscam) and sDscam, we designated these intermediate shortened Dscam genes as $m$ Dscams. As shown in Fig. 1b, nonclassical Dscams dominate the isoform repertoire in all Chelicerata species investigated.

\section{Nonclassical Dscam genes are Chelicerata-conserved and restricted}

This study revealed that nonclassical Dscams are conserved across Chelicerata species and that all types of sDscams (sDscama, sDscam $\beta$, and sDscamy) are largely present in representative species from the 


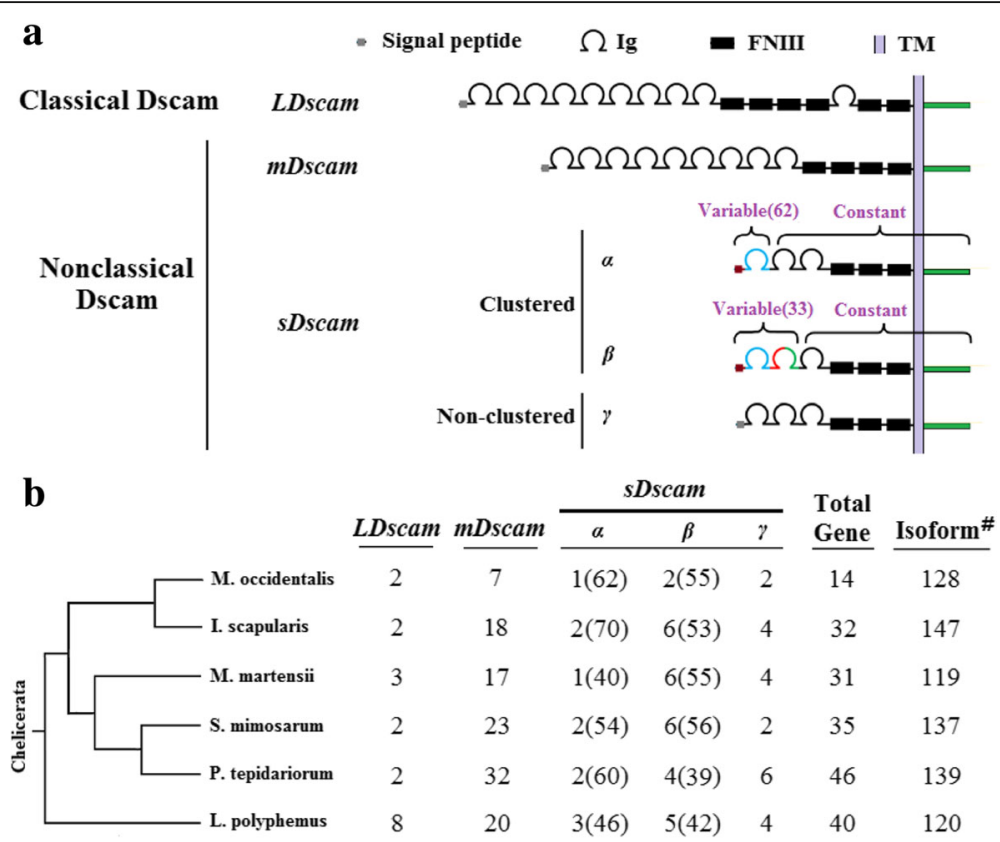

Fig. 1 Genome-wide identification and classification of Dscams in Chelicerata. a Schematic representation of Dscam structures in Chelicerata. Ig, immunoglobulin domains; FNIII, fibronectin III domains. The N-terminal small boxes represent the leader peptides. The lavender blue and green boxes represent the transmembrane (TM) and cytoplasmic domains. Five Dscam types (LDscam, mDscam, sDscama, sDscam $\beta$, and sDscamy) are classified based on their size and structure. LDscam shares structures identical to classical Dscam. mDscam lacks lg10 and FNIII 3-4 domains of classical Dscam. sDscama contains variable $\mathrm{N}$-terminal Igl (blue), which corresponds to the variable lg7 domain of Drosophila Dscam 1. sDscam $\beta$ contains variable $\mathrm{N}$-terminal Ig 1 +2 domains (coloured), which correspond to the variable lg7 + 8 domains of Drosophila Dscam 1. Numbers in parentheses refer to the numbers of 5' variable cassettes in Metaseiulus occidentalis sDscama and sDscam 32 . sDscamy shares domains similar to sDscama and sDscam $\beta$, albeit with no tandemly arrayed cassettes in the $5^{\prime}$ regions. Numbers in parentheses refer to the numbers of tandem cassettes. b Phylogenetic distribution of Dscams and isoform members in chelicerates. Dscams are shown associated with a cladogram of phylogenetic relationships in this study [52]. \# Indicates the putative numbers of Dscam isoforms in various arthropod species caused by either gene and/or exon duplication, estimated by the number of lg7 or orthologues

Arachnida and Merostomata classes investigated in this study (Fig. 1b). Our results suggest that these types of nonclassical Dscams are ancient, existing before the split of Arachnida and Merostomata. The Dscam gene family members diverged markedly across various species. The higher gene number in scorpions, spiders, and horseshoe crabs is consistent with an additional event of whole or large-scale genomic duplications [27]. Although mDscam genes are present in greater numbers than LDscams and sDscams, the sDscam genes can generate up to a hundred isoforms through a combination of alternating promoters and alternative splicing. Thus, based on the isoform number, sDscams are represented more than LDscams and mDscams in each Chelicerata. However, no such nonclassical Dscams have been identified among the Dscam genes from the Mandibulata species of insect, Crustacea, or Myriapoda classes, or in any non-arthropod species. This observation suggests that they arose after the radiation of Mandibulata and Chelicerata during Arthropoda evolution. Thus, we conclude that the nonclassical Dscams are largely conserved and restricted to Chelicerata.

\section{Global analysis of Dscam relationships over the Chelicerata phylogeny}

To trace the evolutionary history of nonclassical Dscam genes, we first performed multiple sequence alignments of all Dscams from six representative species. Comparative analysis of Dscam sequences from Chelicerata and outgroup species revealed three major clades, which represent three groups of LDscam, mDscam, and sDscam (Fig. 2). Based on the phylogenies of individual Dscam types (Additional file 3: Figures S1 and S2), although our analyses of the Dscam genes did not permit a full ancestral reconstruction, several conclusions could be reached. First, these data indicate that at least seven $m D s c a m s$ and five sDscams were present in the Chelicerata ancestor before the split of Arachnida and Merostomata (Additional file 3: Figures S1 and S2). Second, three types of LDscams, mDscams, and sDscams were inconsistently expanded. $m D s c a m$ genes have undergone massive duplications during Chelicerata evolution, while LDscam genes have undergone few or limited duplications (Fig. 2; Additional file 3: Figures S1 and S2). For example, the divergence of the Araneae ancestor into the $P$. tepidariorum and $S$. mimosarum ancestors 


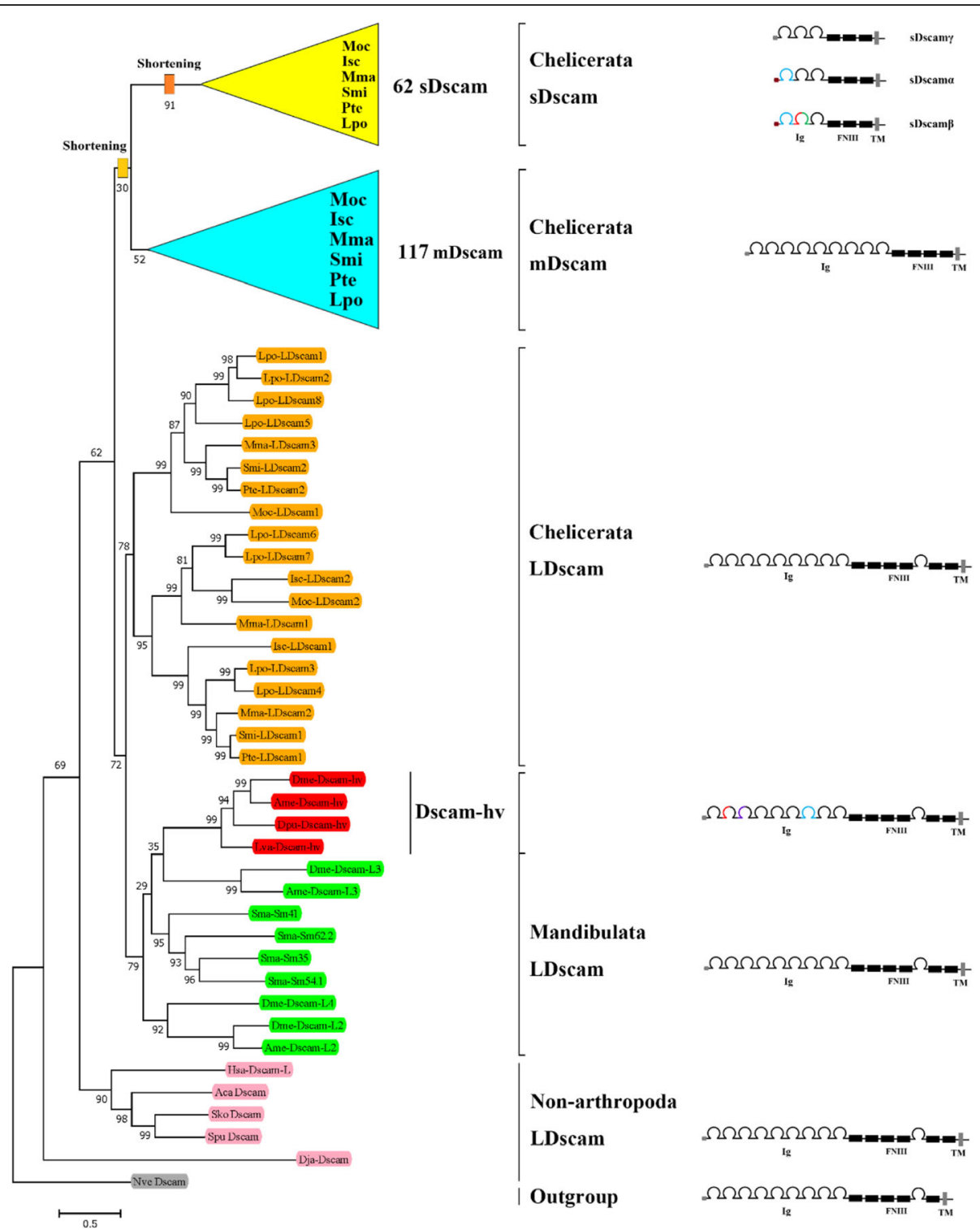

Fig. 2 The evolutionary relationships and protein structures of arthropoda Dscams. The tree is based on amino acid sequence alignment of Dscams encompassing the seventh Ig domains to end (sDscam regions encompassing the first lg domains to end), and is rooted using the sequence of the Nematostella vectensis Dscam (GenBank:ABAV01020293.1) [51]. The support values at the nodes are bootstrap values relative to 1000 replicates. We considered five Dscam types (LDscam, mDscam, sDscama, sDscamß, and sDscamy) in Chelicerates, which are detailed in Additional file 2: Table S2. L. vannamei (Lva) Dscam1 (GQ154653), D. melanogaster (Dme) Dscam1-4 (CG17800; CG42256; CG31 190; CG42330), A. mellifera (Ame) Dscam (AAT96374; BAF03050.1; XM_396307), D. pulex Dscam1 (EU307884), A. californica (Aca) Dscam (ABS30432.1), S. kowalevskii (Sko) Dscam (XP_006825869.1), S. purpuratus (Spu) Dscam (XP_011665747.1), D. japonica (Dja) Dscam (BAE94189.1), and H. sapiens (Hsa) Dscam-L (AAL57166.1) are included. For other canonical Dscam sequences from S. maritima, refer to recent references [24]. We collapsed the monophyletic clades of sDscams and mDscams for visualization convenience. Their detailed phylogenetic relationships are shown in Additional file 3: Figures S1 and S2, respectively. The sequential shortening of the Ig and FNIII domains of canonical Dscam is indicated by orange squares

involved $10 P$. tepidariorum-specific duplications of mDscam (Additional file 3: Figure S1). This gene expansion process is ongoing, as demonstrated by recent duplications in the Araneae species.

Phylogenetic analysis revealed that $s$ Dscams could be clustered into four clades (clades A-D; Additional file 3: Figure S2). Clade D exclusively included $s D s c a m \beta$ s from all species investigated, suggesting this $s D \operatorname{scam} \beta$ is ancient and arose in the Chelicerata ancestor. Clade B consisted of conserved sDscamas and species-specific sDscamy, suggesting that this $s D s c a m \alpha$ arose from the Chelicerata ancestor. Interestingly, clade $C$ included species-specific $s D s c a m \beta$ s and $s D s c a m \alpha s$, in addition to $s D s c a m \gamma$ s. We speculate that an sDscamy ancestor evolved differentially 
into $s D s c a m \beta s$ or $s D s c a m \alpha$ s during Chelicerata divergence. These results indicated that $s D \operatorname{scam} \beta$ s and sDscamas might have multiple independent origins.

\section{A lineage-specific burst of $5^{\prime}$ clustered cassettes in sDscam genes}

To produce an overview of the evolutionary relationships among the variable cassettes, we generated heatmaps for sDscam $\alpha$ and $s D \operatorname{scam} \beta$ to show the relative similarities of each cassette repeat to other variable repeats both within and between species. For these analyses, we selected one representative species from each of the major orders investigated: $M$. occidentalis, S. mimosarum, $M$. martensii, I. scapularis, and L. polyphemus. Analysis of the heatmaps of the tandemly arrayed $5^{\prime}$ cassettes in the sDscamas and $s D s c a m \beta$ s revealed little evidence of conserved orthologous pairs of repeats between species (Fig. 3a, b). Instead, when striking similarities were found between repeats in each species, they typically involved large blocks of highly similar cassettes within each gene of one species. For example, a massive block of 62, 36, and 18 cassettes expanded specifically in sDscama of M. occidentalis, and in $s D s c a m \alpha 1$ and $s D s c a m \alpha 2$ of $S$. mimosarum, respectively (Fig. 3c). Similarly, blocks of 21 and 33 cassettes in the $M$. occidentalis $s D$ scam $\beta 1$ and $s D s c a m \beta 2$, respectively, were highly similar to one another (Fig. 3b, d). Phylogenetic analysis of duplicated cassettes from two closely related spiders (S. mimosarum and P. tepidariorum) indicated that $5^{\prime}$ cassette duplication largely occurs in a lineagespecific manner (Additional file 3: Figure S3). Furthermore, duplicated cassettes showed the tendency to be located adjacent to one another within a gene. Overall, these data indicate that the main expansions of ancestral cassettes of $s D$ scam $\alpha$ and $s D s c a m \beta$ s occurred independently in each lineage.

The heatmap patterns differed considerably between $s D s c a m \alpha$ s and $s D s c a m \beta s$ among the various species. The sDscama heatmap revealed large blocks of cassette duplications specific to each clade, consistent with phylogenetic analyses of duplicated cassettes across Chelicerata species (Fig. 3c). In contrast, with the exceptions of M. occidentalis, most $s D s c a m \beta$ s contain a number of common blocks that span multiple clades, as represented in green shading in Fig. 3b. Notably, duplications of $5^{\prime}$ cassettes in sDscamas and $s D s c a m \beta s$ are almost exclusively specific to $M$. occidentalis, showing fewer similarities with other cassettes from other species, as represented by blue blocks in the regions of the heatmap that compare two species (Fig. 3a, b). Such high species specificity of the $5^{\prime}$ variable cassettes suggests that $s D s c a m \alpha$ and $s D s c a m \beta$ s have undergone rapid expansions and divergence. Furthermore, comparison of $5^{\prime}$ cassette- and gene-based clustering clearly indicated that $5^{\prime}$ cassette duplication occurs on a much faster timescale than gene duplication (Additional file 3: Figure S4). This multi- layer expansion of sDscam diversity might help to increase the efficiency and flexibility of spatiotemporal regulation.

\section{5 ' cassette duplicate inversely correlated with sDscam gene duplicates}

As gene duplications and $5^{\prime}$ cassette tandem duplications increased isoform diversity through the emergence of additional genomic copies, we next investigated whether or how they are related to each other as evolutionary mechanisms. We found that the number of $5^{\prime}$ cassette tandem duplicates correlated inversely with that of gene duplicates in the $s D$ scam $\alpha$ and $s D s c a m \beta$ subfamily (Fig. 4a, b). Single sDscams (singletons) are likely to contain substantially more tandem cassettes in the $5^{\prime}$ variable region. For example, up to 62 and 40 copies reside in the $5^{\prime}$ variable region of sDscama in M. occidentalis and M. martensii, respectively. Furthermore, the sDscam $\alpha$ subfamily (1-3 members) contains two- to four-fold more $5^{\prime}$ cassette duplicates per gene, which is similar to members in the larger $s D$ scam $\beta$ subfamily (2-7 members) (Fig. 4c); we found the reverse trend in gene duplicates (Fig. 4d). Importantly, the total number of Dscam isoforms is roughly similar among various Chelicerata species (Fig. 1b). Therefore, this correlation may reflect compensatory evolution between alternative promoter and gene duplication, analogous to the inverse correlation of alternative splicing with gene duplication [28]. These results imply that the inverse correlation is not simply an inherent inclination, but is instead fulfilling the complementary demand for expanding Dscam isoforms via distinct evolutionary mechanisms.

To further examine the evolutionary forces underlying the inverse correlation, we carried out a detailed comparison of duplication scenarios in sDscam $\alpha$ and Dscam $\beta$ genes from various species. Interestingly, the number of $5^{\prime}$ cassette tandem duplicates per gene correlated inversely with the size of the duplicate blocks in M. occidentalis, $I$. scapularis, M. martensii, and S. mimosarum (Fig. 4e-h). These correlations largely fitted to a power law. $L$. polyphemus did not exhibit as strong a correlation as the other species (Fig. 4i), possibly due to the incomplete annotation of the Dscam genes. Furthermore, the numbers of $5^{\prime}$ cassette duplicates per sDscama were roughly 1-3fold higher than those in $s D s c a m \beta s$, with the duplicate blocks containing two additional exons than those in sDscamas (Fig. 4j). This inverse relationship might reflect an inherent property of the species, i.e., the genome size (Fig. 4k, l). For example, I. scapularis, with a large genome size estimated at $2100 \mathrm{Mb}$ [29], contained more sDscam gene duplicates, but fewer $5^{\prime}$ cassette tandem duplicates in each gene. In contrast, $M$. occidentalis, with a small genome size $(\sim 152 \mathrm{Mb})$, had fewer sDscam gene duplicates, but more $5^{\prime}$ cassette tandem duplicates per gene. In particular, phylogenetic analysis indicated loss of cassettewithin introns occurred independently in $s D \operatorname{scam} \beta 1$ and 

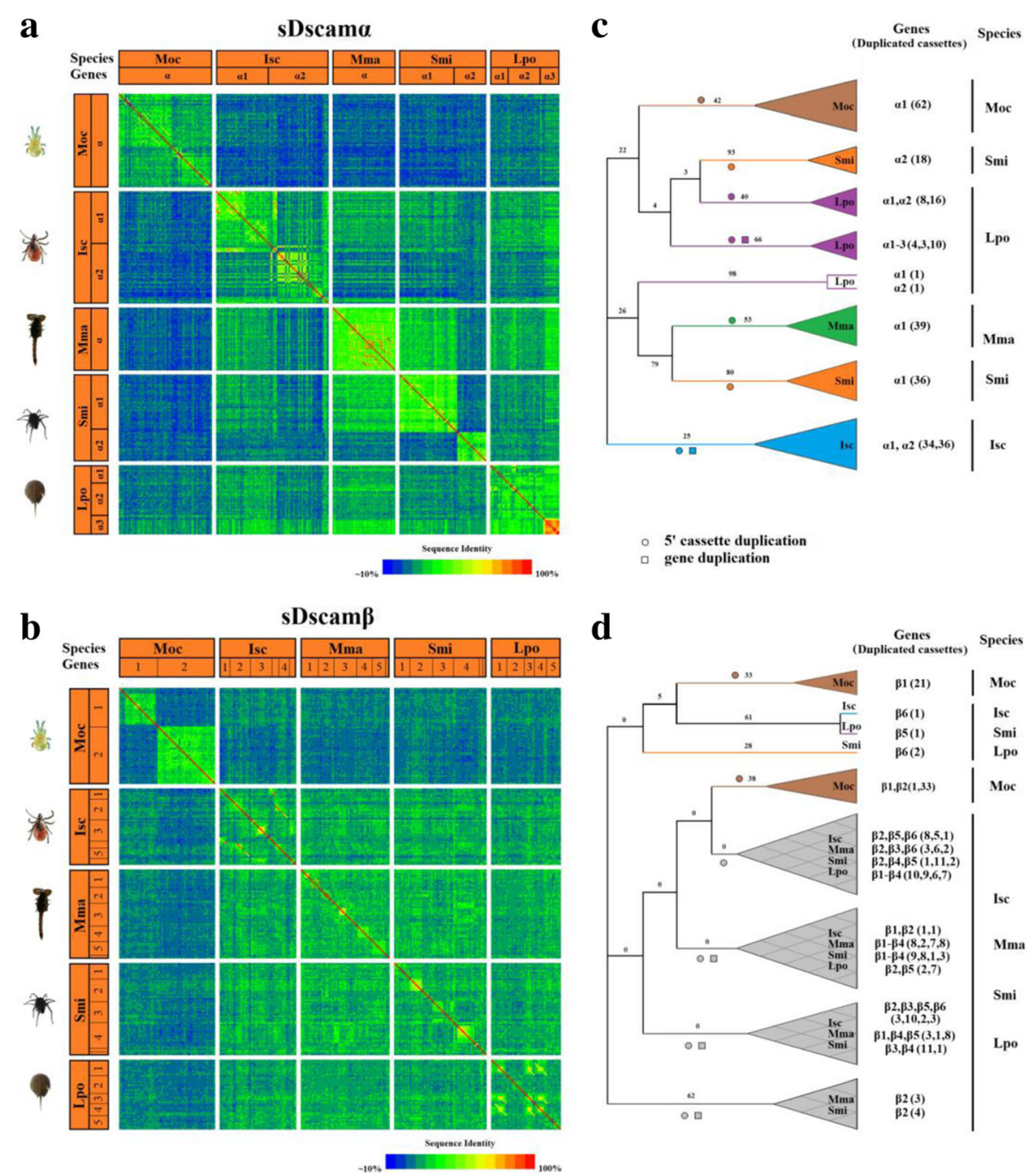

Fig. 3 Similarities among 5' variable cassettes of sDscam genes in representative species from each of the major Chelicerata clades. a Heatmap of pairwise sequence identities of the duplicated cassettes encoding the Ig1 domain of sDscama isoforms from Metaseiulus occidentalis (Moc), Ixodes scapularis (Isc), Mesobuthus martensii (Mma), Stegodyphus mimosarum (Smi), and Limulus polyphemus (Lpo). To simplify visualisation, the cassette repeats are depicted in the heatmaps in the same linear order in which they reside in each gene. $\mathbf{b}$ Heatmaps of the percent identities of the duplicated cassettes encoding $\lg 1+2$ domains of sDscam $\beta$ in Chelicerata species. The order of the cassettes in the heatmap corresponds to the linear order of each cassette in the genome. c Phylogenetic tree for the 268 duplicated cassettes encoding Ig 1 domains of sDscama. The support values at the nodes are bootstrap values relative to 1000 replicates. Numbers in parentheses refer to the number of $5^{\prime}$ variable cassettes of the corresponding gene. Clades were collapsed for visualisation convenience. $\mathbf{d}$ Phylogenetic tree for the 243 duplicated cassettes encoding Ig $1+2$ domains of sDscam $\beta$. Collapsed clades plus crosses are depicted as a combined clade composed of these duplicated cassettes from different species

sDscam $\beta 2$ of $M$. occidentalis, whereas no substantial intron loss occurred in the constant region (Fig. $4 \mathrm{~m}$ ). This result led us to speculate that intron loss caused the decreased repeat block size to facilitate greater duplication in the $5^{\prime}$ variable region. Conversely, this species-specific intron loss might be driven by the selection pressure of greater cassette duplication. These results suggest that gene duplications and $5^{\prime}$ cassette tandem duplication are not selected to expand independently of each other during Chelicerata evolution.

\section{Differential and biased expression of nonclassical Dscam isoforms}

To further characterise the expression of nonclassical Dscam isoforms, we employed RNA-seq data to analyse the expression of the nonclassical Dscams in various tissues of $M$. martensii. Similar to $s D s c a m \alpha$ and $s D s c a m \beta$ [25], as well as three classical Dscams (LDscams1-3), 14 of $17 \mathrm{mDscams}$ and 3 of $4 \mathrm{sDscamys}$ were expressed at markedly higher levels in the cephalothorax than in other tissues (Fig. 5a; Additional file 3: Figure S5). This expression pattern is largely coincident with high expression of 


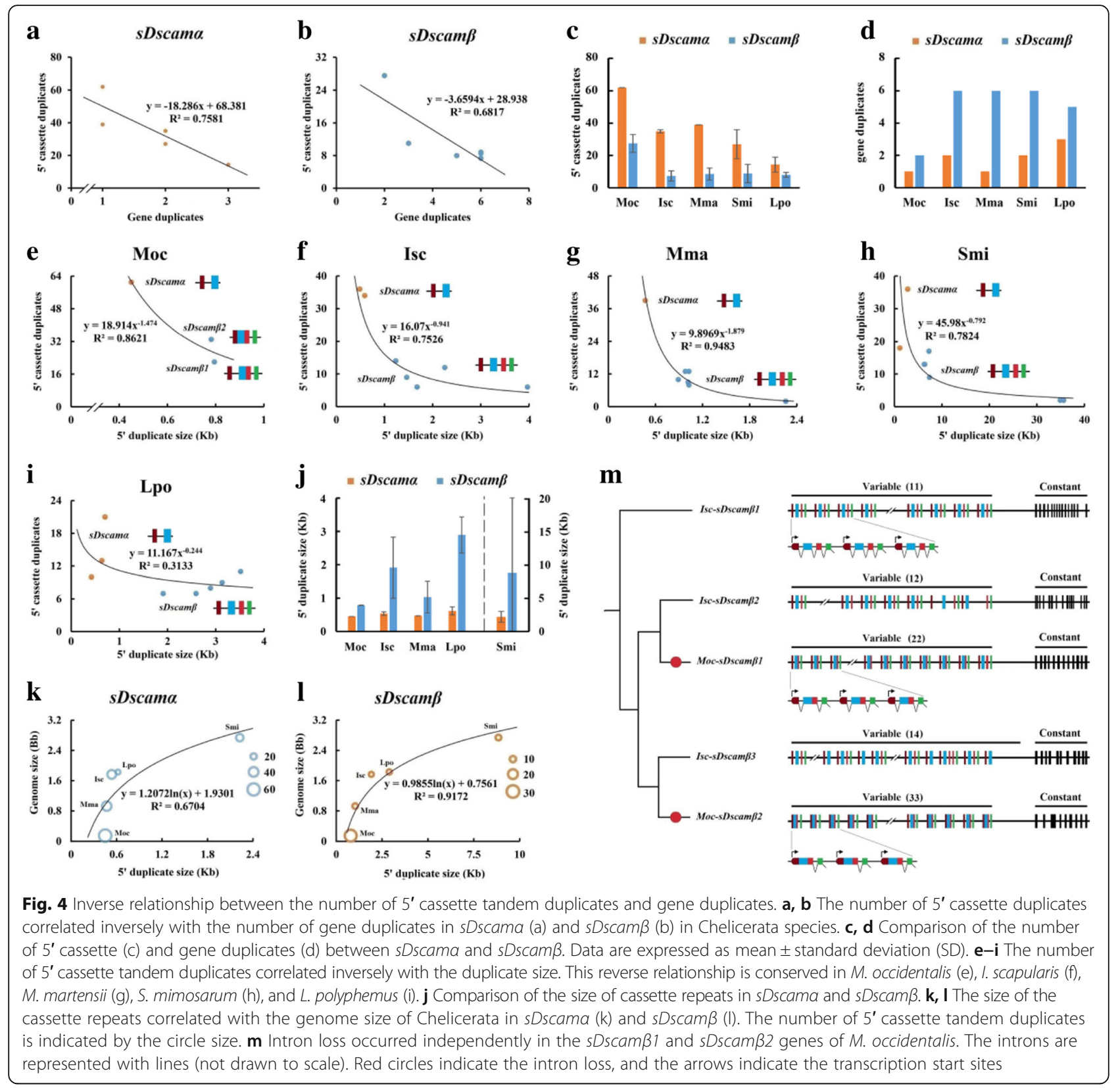

classical Dscams in the nervous system of vertebrates and insects [5, 30-32]. In contrast, LDscams, mDscams, and sDscams showed low expression in muscles. Notably, $m D s c a m 8$ was specifically expressed at maximum levels in haemocytes, whereas $m D s c a m 10$ and $m D$ scam15 were highly expressed in poison glands (Fig. 5a). It will be interesting to see whether nonclassical Dscams play a potential role in Scorpione immunity, similar to that of Dscam1 isoforms in the Drosophila immune system [5]. These results suggest that nonclassical Dscam expression is regulated spatially and temporally.

The wealth of published RNA-seq data from a set of 20 embryo-derived cell lines allows the comparison of
Dscam expression in various cell lines of $I$. scapularis (Additional file 4: Table S3). Expression profiling revealed that Dscam genes were differentially expressed in various cell lines (Fig. 5b). Interestingly, many Dscams were preferentially and specifically expressed in certain lines. For example, high expression of mDscam14 was observed in strain IDE2, but was almost undetectable in strain IDE8 and ISE18. In contrast, sDscamy4 showed robust expression in strain IDE8 and ISE18, but low level expression in strain IDE12; sDscam $\beta 2$ showed high expression in strain ISE18. Likewise, the $5^{\prime}$ variable exons of sDscam $\alpha$ and $s D s c a m \beta$ genes exhibited differential expression in various lineages (Additional file 3: 

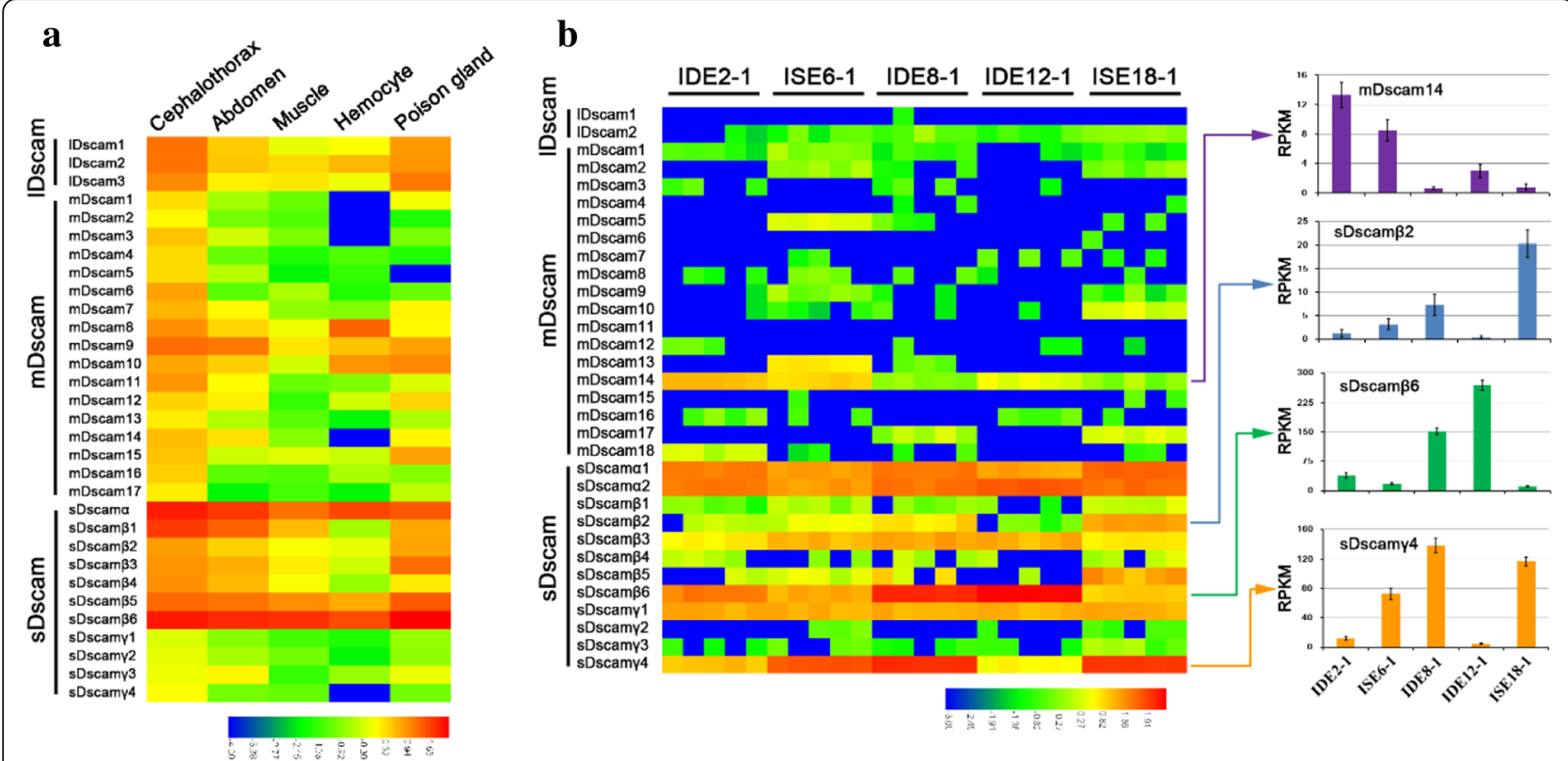

Fig. 5 Differential expression of nonclassical Dscam genes. a Heatmap of expression of 31 Dscam genes in various tissues of M. martensii. The expression level for each transcript is shown as reads per kilobase of transcript per million mapped reads (RPKM) of its corresponding constitutive exons. The 25-nt fragmented RNA-sequencing datasets were mapped to calculate the relative expression level. The maximum expression levels of mDscam8 were found in hemocytes, whereas mDscam 10 and mDscam 15 were highly expressed in poison glands. $\mathbf{b}$ The differing expression patterns of Dscams in I. scapularis lineages. This result indicates that many Dscams were preferentially and specifically expressed in certain lines. Data are expressed as the mean \pm SD from three independent experiments

Figure S6). sDscama2.14 was specifically expressed at maximum levels in strain ISE6, while $s D s c a m \beta 2.12$ and $s D$ scam $\beta 6.6$ were expressed at maximum levels in strain ISE18 and IDE12, respectively. Collectively, these results revealed lineage-specific expression signatures of Dscam isoforms.

We observed a dramatic bias: sDscams were largely expressed at higher levels than LDscams and mDscams in various tissues of $M$. martensii (Fig. 6a), and in different I. scapularis lineages (Fig. 6b). A similar bias was observed in S. mimosarum, $P$. tepidariorum, $M$. occidentalis, and L. polyphemus (Fig. 6c). These data indicate that $s D s c a m$-biased expression patterns are evolutionally conserved across Chelicerata. It is possible that the high expression of $s D s c a m s$ might be due to the use of multiple promoters, leading to more transcripts. Taken together, both the number and expression level of nonclassical Dscams dominate exclusively in the Dscam isoform repertoire across Chelicerata.

\section{Discussion}

\section{The evolutionary landscape of Dscam diversity in} arthropods

This study identified large and diverse nonclassical Dscam repertoires in chelicerates, most of which are newly annotated or corrected. The results reported here extend our previous insights into Dscam diversity during arthropod evolution [23-25]. Firstly, the nonclassical Dscam genes can be classified into four types $(m D s c a m$, sDscama, sDscam $\beta$, and $s D s c a m \gamma$ ) based on their size and structure (Fig. 1). Moreover, the nonclassical Dscam genes are conserved and restricted to Chelicerata, suggesting that Chelicerata uniquely evolved a large lineage-specific repertoire of nonclassical Dscam isoforms. Phylogenetic analysis and comparison of Dscam structures revealed that the Dscam ancestor underwent multiple shortening events during chelicerate evolution, leading to the loss of protein domains to varying degrees [25]. Thus, the Dscam gene number has undergone massive expansion, and also the structure of Dscam genes became highly diversified during Chelicerata evolution. These findings, together with those of others [23-25], provide a global framework for the evolution of Dscam diversity in arthropods.

These Dscam diversifications suggest that their binding mechanisms are potentially different from those of classical Dscams. The crystal structural analysis reveals that the first four Ig domains of Drosophila Dscam1 are responsible for the formation of the horseshoe configuration [33]. Additional modelling studies illuminated the molecular basis of the isoform-specific homophilic binding specificity [9]. As they contain the same eight $\mathrm{N}$ terminal Ig domains as Drosophila Dscam1, it is conceivable that the chelicerate mDscams studied in this 


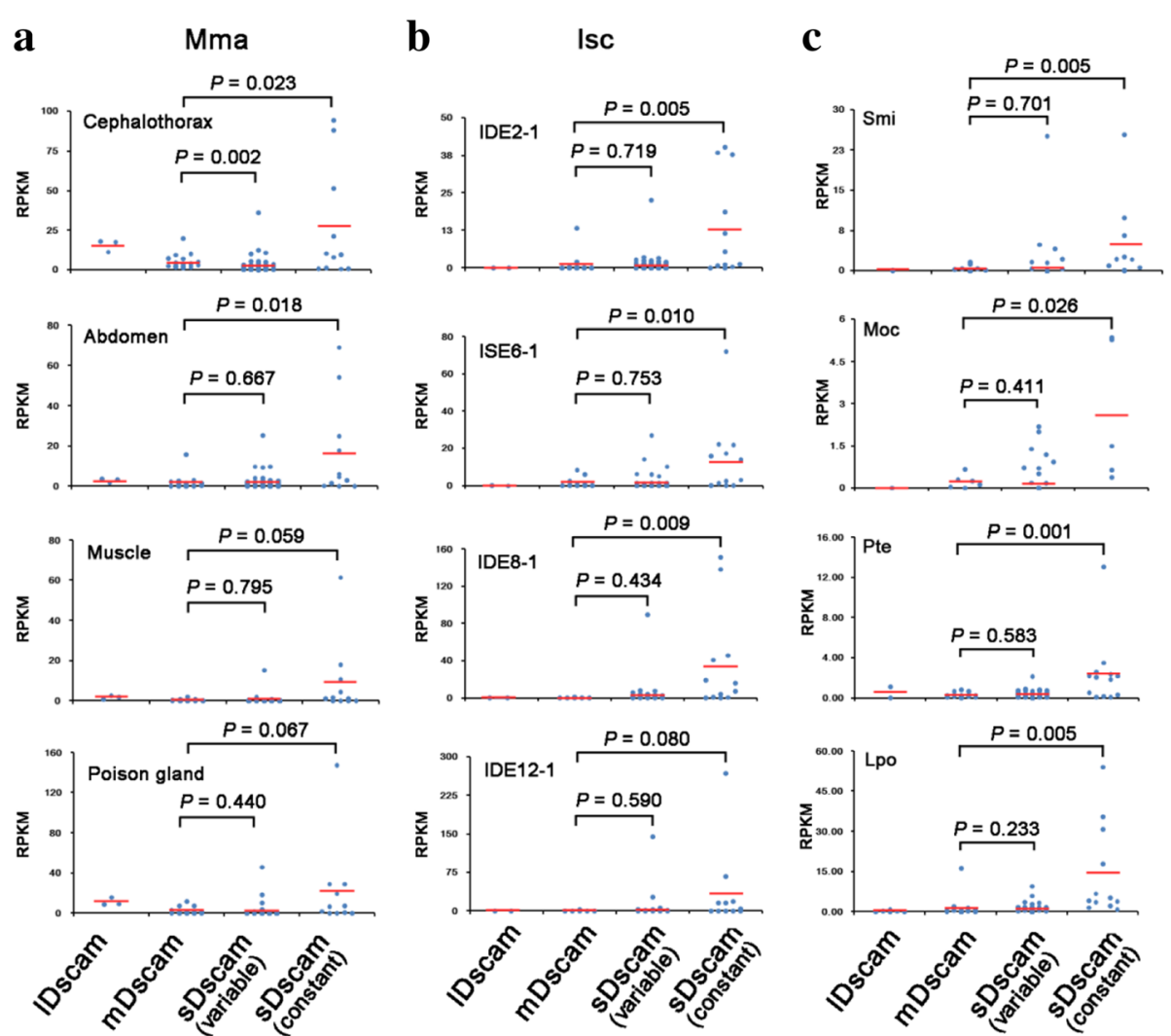

Fig. $6 \mathrm{sDscam}$ genes are biased to be more highly expressed. a In M. martensii, sDscam genes are biased to be more highly expressed than mDscam genes. We calculated $p$ values using a two-tailed Student's t-test. As LDscams occurs much less frequently than mDscams and sDscams, we did not analyze the statistical differences between them. $\mathbf{b}$ sDscam genes are biased to be more highly expressed than $\mathrm{mDscam}$ genes in $\mathrm{I}$. scapularis. c The expression bias is conserved in S. mimosarum (Smi, SRR1015314), P. tepidariorum (Pte, SRR1824487), M. occidentalis (Moc, SRR446504), and L. polyphemus (Lpo, SRX1323743)

research could form a horseshoe configuration and interact via a similar mechanism. However, this horseshoe structure does not form in chelicerate sDscams with only three Ig domains. Therefore, we speculate that chelicerate sDscams exhibit a different mode of isoformspecific homophilic binding than Drosophila Dscam1.

Chelicerata seem to have generated far fewer Dscam isoforms than insects. As estimated by the number of Ig7 or orthologues, the number of Dscam isoforms is in the range of $\sim 100-200$ across the Chelicerata species investigated (Fig. 1), approximately two to three orders of magnitude lower than that in insects. Recent studies indicate that clustered mammalian Pcdhs could expand the binding specificity repertoire via cis-multimers. For 22 $\gamma$-Pcdhs, the diversity of adhesive interfaces could be on the order of $10^{5}$ through cis-tetramerisation coupled with homophilic trans interactions [34, 35]. Given the striking organisational resemblance between the Chelicerata clustered $s$ Dscams and mammalian Pcdhs, it is attractive to speculate that Chelicerata sDscams could function via cis-multimers. If so, the diversity of adhesive interfaces mediated by Chelicerata sDscams will be much higher, as there are many more Chelicerata-clustered $s D s c a m s$ (90-130) than mammalian-clustered Pcdhs (50-60).

\section{Dscams versus Protocadherins}

Dscams and Pcdhs belong to large established families of cell adhesion molecules: Dscams belong to the Ig superfamily and Pcdhs belong to the cadherin superfamily [36]. The clustered Pcdh diversity is confined within the clade of jawed vertebrates, which is considered as a chordate innovation [37], whereas extensive Dscam diversity is unique to arthropods. In the latter case, arthropods diversify using two mechanisms to generate Dscam isoform diversity: Mandibulata Dscam genes employ exclusive splicing of internal exon clusters to generate distinct isoforms [3, 24] and chelicerate Dscams utilise alternative promoters in the $5^{\prime}$ variable region [25]. However, neither cadherin nor protocadherin genes appear to have internal tandem exon arrays in a manner similar to Mandibulata Dscams.

Both clustered chelicerate Dscams and vertebrate Pcdhs are organised in a tandem array in the $5^{\prime}$ variable region (Fig. 7a, b). Curiously, both $5^{\prime}$ clustered sDscams 
and Pcdhs appeared to originate via an analogous evolutionary pathway (Fig. 7c, d), which was involved in the shortening and expansion of Dscam and cadherin ancestors $[25,38]$. In both genes, each variable repeat is preceded by a promoter, and differential expression occurs via combining alternate promoter choice with alternative splicing [19, 20, 25]. Moreover, 5' clustered Dscams and Pcdhs contain similar structural composition encoding six extracellular domains, a single transmembrane (TM) region, and a cytoplasmic domain. Interestingly, threedimensional protein structure modelling revealed a simi$\operatorname{lar} \beta$ sandwich structure between the first domain of Pcdh and sDscam (Fig. 7a, b). Finally, we showed that clustered sDscams encode proteins exhibiting isoformspecific homophilic binding in a manner similar to Pcdhs. Despite their overall similarities, the structural properties of $P c d h$ and $s D s c a m$ genes differ in at least two major aspects. First, inconsistent with the conserved arrangement of a single genome locus containing three tandem Pcdh gene clusters across vertebrates, the sDscams are largely dispersed or partially clustered in the chelicerate genome. As the transcription of $P c d h$ gene clusters is closely linked and mediated by longrange chromatin-looping interactions [39], the dispersed distribution of sDscam gene clusters might reflect a mode of transcription regulation distinct from that of Pcdh genes. A second major difference concerns the structure of the variable region. Pcdh variable exons encode the entire ectodomain composed of six extracellular cadherin domains (EC1-EC6), a single TM region, and a short cytoplasmic extension, whereas the variable cassettes of sDscams encode the partial ectodomain of one or two Ig domains at the $\mathrm{N}$-terminus. Future studies are needed to investigate the role of variable region structures in the subcellular distribution, isoformspecific binding, and multimer formation.

Combining experimental evidence with the complementary phylogenetic distribution of Dscam diversity in arthropods and Pcdh diversity in vertebrates, it is tempting to suggest that both may have similar roles in the nervous system [15-17, 21, 22]. These two phyla seem to employ a similar strategy for self/non-self discrimination by recruiting various molecules of different protein families [15]. Nevertheless, there is a wide evolutionary gap between arthropods and vertebrates, as they share a common ancestor more than 500 million years ago. It will be informative to explore the molecules or mechanisms of species within this phylogenetic gap (i.e., Branchiostoma floridae), which are thought to lack both clustered Pcdh and Dscam genes, and that have evolved

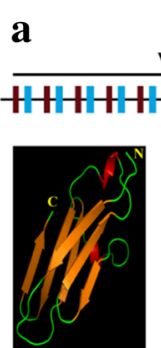

Ig1
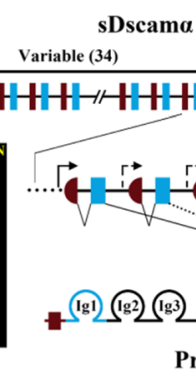

c

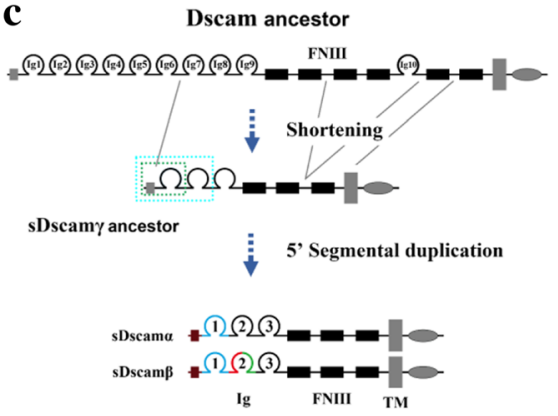

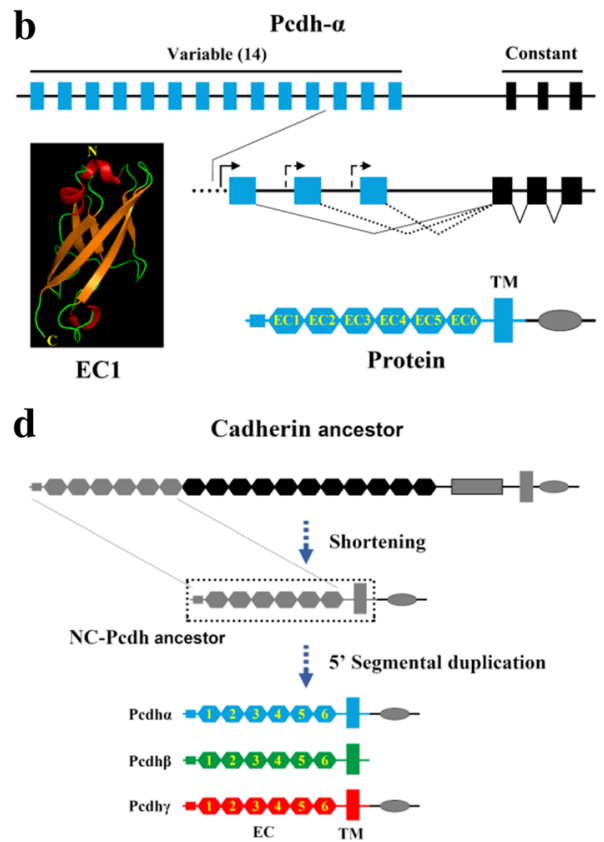

Fig. 7 Comparisons of organisation and origin of clustered Dscam and Pcdh genes. a Schematic diagram for the sDscama gene in Chelicerata. Symbols used are the same as in Fig. 1. Each variable cassette was transcribed by an alternative promoter followed by alternative splicing. The variable cassette encoded the N-terminal Ig1 domain (blue). Tertiary structure model of Ig1 of I. scapularis sDscam is shown on the left. b Schematic diagram for the Pcdha genes in vertebrates. The Pcdh gene cluster contains exons that encode 14 extracellular and TM domains [18]. Each repeat is preceded by a promoter, and encodes extracellular and TM domains. Tertiary structure model of the EC1 domain of Pcdha is shown on the left, which is similar to that of the Ig1 domain of sDscam. $\mathbf{c}$, $\mathbf{d}$ Comparisons between the evolutionary origin and expansion of clustered Dscam (c) and Pcdh (d) genes. The $5^{\prime}$ clustered organisation of both sDscam and Pcdh genes may originate from the shortening and expansion of the ectodomains of canonical Dscam via sequential duplication and mutation 
to endow cells with distinct molecular identities and highly diverse recognition selectivity strategies.

\section{Diversification of lg superfamily protein repertoires}

Although tandemly arrayed Ig repeats are found frequently in the genome, animals achieve protein diversity from a single locus via a variety of mechanisms (Additional file 3: Figure S7). In higher vertebrates, the great diversity of antigen-specific receptors of the adaptive immune system can be achieved through somatic gene rearrangement and clonal selection at the DNA transcriptional level, which is known as the V(D)J mechanism [40-43]. However, insect Dscam1s utilise mutually exclusive splicing to generate an extensive repertoire of thousands of Ig-superfamily protein isoforms $[3,5]$. In contrast, our studies indicate that Chelicerata $s D s c a m s$ employ alternative promoters to generate substantial numbers of isoforms [25]. These molecular processes are mutually exclusive among three distinct clades, functioning to create extensive molecule diversity. Metazoans might have evolved different ways for extensive Ig-superfamily proteins to enable immune defence and other functions.

\section{Conclusions}

In this study, we identified large and lineage-specific nonclassical Dscam repertoires in chelicerates. Nonclassical Dscams are conserved and restricted to Chelicerata, and have been classified into four types based on their size and structure. These results demonstrate that arthropods specifically diversify a large Chelicerata-specific repertoire of nonclassical Dscam isoforms. The Dscam isoform repertoire across Chelicerata is dominated exclusively by the number and expression levels of nonclassical Dscams. This genome-wide identification and classification study of Dscam genes provides the global framework of the evolution of Dscam diversity in arthropods, and provides mechanistic insights into the diversification of the species-specific Ig superfamily repertoire.

\section{Methods}

\section{Data availability and RNA-seq data analysis}

We investigated the following representative Chelicerate species: Mesostigmatan M. occidentalis, Trombidiformes I. scapularis [29], two Araneae S. mimosarum and P. tepidariorum [44], two Scorpiones M. martensii [45], and Merostomatan L. polyphemus. The sources of the Chelicerata genome sequences used in this study are shown in Additional file 1: Table S1. To validate the Dscam candidates, we selected 125 publically available RNA-seq datasets corresponding to various developmental stages, tissues, organs, and cell lines across six chelicerate species (Additional file 4: Table S3). All of the raw RNA-seq datasets were subject to pre-treatment, including adapter trimming and low-quality read removal using the FASTX-Toolkit (http://hannonlab.cshl.edu/fastx_toolkit/index.html). Then, for each sequencing dataset, the count of each RNA-seq read was normalized to reads per million (RPM), thus enabling cross-sample comparison of the Dscam expression levels. Specifically, to calculate the RPM of one RNA-seq read, the raw count of the read was divided by the total raw count of the RNA-seq dataset and multiplied by $10^{6}$. The treated reads were mapped onto the transcripts using Bowtie 2 [46]. From these mappings, we were able to calculate the expression levels in reads per kilobase of transcript per million mapped reads (RPKM).

\section{Availability of genome and RNA-seq data}

We investigated the following representative species of Chelicerate: Mesostigmatan M. occidentalis, Trombidiformes I. scapularis [29], two Araneae S. mimosarum and P. tepidariorum [44], two Scorpiones M. martensii [45], and Merostomatan L. polyphemus. The sources of the Chelicerata genome sequences used in this study are shown in Additional file 1: Table S1. For Dscam candidate validation, we selected 125 publically available RNA-seq data corresponding to various developmental stages, tissues, and organs, and cell lines across six chelicerate species (Additional file 4: Table S3).

\section{Annotation and identification of Dscam genes}

The sequences of the Dscam homologues were annotated through cross-species BLAST searches using the available annotated Dscam sequences (http://blast.ncbi.nlm.nih.gov/Blast.cgi). These Dscam candidate homologues were validated further using publically available transcriptome and RNA-seq datasets. All Dscam candidates were confirmed by phylogenetic analysis (http://www.ebi.ac.uk/ clustalw/index.html) and then analysed by classifying and predicting protein domains with InterPro [47] (http:// www.ebi.ac.uk/interpro/) and PROSITE [48] (http://prosite.expasy.org/prosite.html). The Dscam genes identified in representative species of Chelicerate are listed in Additional file 2: Table S2.

\section{Phylogenetic analysis}

The Dscam sequences were aligned across species using the Clustal W2 software package (http://www.ebi.ac.uk/ clustalw/index.html) [49]. The coding sequences of the variable region were translated, and the resulting polypeptides were aligned. The nucleotide sequences of each $5^{\prime}$ variable cassette of sDscam were translated into amino acid sequences and aligned. The genetic distances for each gene were estimated using MEGA 7.0 software [50]. We used maximum likelihood (ML) methods, using MEGA [50], to build the phylogenies. For the ML analysis, we ran MEGA with at least 1000 bootstrap replicates. To determine the homology of the Dscam-related 
genes found in metazoans, we estimated the phylogenies of 217 proteins, including Dscams encompassing the seventh Ig domains to the end (sDscam regions encompassing the first Ig domains to end) (Fig. 2). This phylogeny was rooted using the sequence of the Nematostella vectensis Dscam (GenBank:ABAV01020293.1) [51]. We used the duplicated cassettes encoding the Ig1 domain of sDscam $\alpha$ to dissect the evolutionary relationships between the duplicated cassettes with a tree rooted on the $D$. melanogaster Dscam1 duplicated exon 9.1, which encodes the Ig7 domain (Additional file 3: Figure S3a). Similarly, we used the duplicated cassettes, which encode the Ig1 +2 domain of $s D s c a m \beta$ and a tree rooted on the $D$. melanogaster Dscam1 exon 9.1, 10, and 11, which encode the Ig7 + 8 domains (Additional file 3: Figure S3b).

\section{Protein three-dimensional structure modelling}

All three-dimensional proteins structures were acquired using the Swiss-Model (automated mode) (www.swissmodel.expasy.org). The structures were displayed and processed using the PyMOL software package (www.pymol.com).

\section{Analysis of differential and biased expression}

The RNA-seq data from publically available samples were used to analyse the expression of nonclassical Dscam genes at various developmental stages, tissues types, and cell lines (Additional file 4: Table S3). For each sample, we calculated the RPKM value of the constant exonic region to measure the expression level of each Dscam gene from the replicates. The alternative exon encoding Ig7 was selected to calculate the expression level from the replicates for each $5^{\prime}$ variable cassette. Considering the short length of the alternative exons, the RNA-seq reads were split into 25-nucleotide (nt) fragments for mapping by using Bowtie 2 software [46], and only the perfectly mapped fragments were retained for expression level calculation. Furthermore, the read counts of a 25-nt fragment with multiple loci were divided by the number of loci, and then assigned equally to each locus for expression level calculation. To eliminate influences on calculations of the expression levels from identical sequences among exon duplicates, both the 25-nt fragments and the full-length RNA-seq reads (150 nt) were used to calculate the expression profiles as previously described [25]. If, as a result of originating from a repetitive region, one RNA-seq read or fragment mapped onto several loci, we divided the RPM value of this RNA-seq read by the number of repetitive loci, then evenly assigned it to each transcript in the expression level calculation.

\section{Statistical analysis}

We used an independent sample $t$-test to assess the relationship between the $5^{\prime}$ cassette duplicate and the
sDscam gene duplicate. We compared the numbers of $5^{\prime}$ cassettes and gene duplicates between the sDscam $\alpha$ and sDscam $\beta$ groups using a two-tailed Student's $t$-test. We defined correlation and significance levels for the number of $5^{\prime}$ cassette duplicates and the repeat size in terms of a simple regression model. We also used a twotailed Student's $t$-test to compare the differences between the expression levels in groups of $\mathrm{mDscams}$ and sDscams across various species. Effects were considered to be statistically significant when $p<0.05$.

\section{Additional files}

Additional file 1: Table S1. Chelicerata species and their genome sources in this study. (XLSX $10 \mathrm{~kb}$ )

Additional file 2: Table S2. A list of Dscam homologues identified in Chelicerata species. (XLSX $25 \mathrm{~kb}$ )

Additional file 3: Supplementary Figures S1-S7. (PDF 1532 kb)

Additional file 4: Table S3. RNA-seq datasets in Chelicerata species. (XLSX $21 \mathrm{~kb})$

\section{Abbreviations \\ BLAST: Basic local alignment search tool; Dscam: Down syndrome cell adhesion molecule; EC: Extracellular cadherin domains; FNIII: Fibronectin III; Ig: Immunoglobulin; Isc: Ixodes scapularis; LDscam: Large Dscam; Lpo: Limulus polyphemus; mDscam: Middle Dscam; MEGA: Molecular evolutionary genetics analysis; Mma: Mesobuthus martensii; Moc: Metaseiulus occidentalis; mRNA: Messenger RNA; Pcdhs: Protocadherins; Pte: Parasteatoda tepidariorum; RNA-seq: RNA Sequencing; RPKM: Reads per kilobase of transcript per million mapped reads; RPM: Reads per million; \\ sDscam: Shortened Dscam; Smi: Stegodyphus mimosarum; \\ TM: Transmembrane}

\section{Acknowledgments}

We thank Dr. Qi Zhou, Institute of Life Sciences, Zhejiang University, China, for the critical reading of the manuscript.

\section{Funding}

This work was supported by research grants from the National Natural Science Foundation of China (31630089, 31430050, 91740104).

\section{Availability of data and materials}

All data generated or analysed during this study are included in this published article and its Additional files. The Dscam gene sequences were deposited into GenBank with accession numbers: MF106020-MF106053; MF066898-MF066905; KX555546-KX555560; KU641421- KU641424.

\section{Authors' contributions}

YJ conceived of this project. GC, YS, HL, JZ, HM, HD, SC and YW annotated and analyzed the sequences; YJ, GC and SH designed the experiments; $\mathrm{SH}$, GC, PG and WH cloned the nucleotide sequences; YS and IZ conducted phylogenetic and evolutionary analysis; SH and GC conducted alternative splicing analyses; SH and YM analyzed exon junction; YM and GC performed performed the computational analysis of expression level; GC and JZ analyzed promoter activity. YJ and YM performed data correlations. YJ, SH, GC, YM, YS, BX and FS analyzed the data; YJ and YM wrote the manuscript; all authors discussed the results and commented on the manuscript.

Ethics approval and consent to participate Not applicable.

Consent for publication Not applicable. 


\section{Competing interests}

The authors declare that they have no competing interests.

\section{Publisher's Note}

Springer Nature remains neutral with regard to jurisdictional claims in published maps and institutional affiliations.

\section{Author details}

'Zhejiang University, Institute of Biochemistry, College of Life Sciences, Hangzhou, Zhejiang ZJ310058, People's Republic of China. ${ }^{2}$ College of Life and Environmental Sciences, Hangzhou Normal University, Hangzhou, Zhejiang ZJ310018, People's Republic of China.

Received: 10 August 2017 Accepted: 22 December 2017 Published online: 19 January 2018

\section{References}

1. Nilsen TW, Graveley BR. Expansion of the eukaryotic proteome by alternative splicing. Nature 2010;463:457-463.

2. Keren H, Lev-Maor G, Ast G. Alternative splicing and evolution: diversification, exon definition and function. Nat Rev Genet. 2010;11:345-55.

3. Schmucker D, Clemens JC, Shu H, Worby CA, Xiao J, Muda M, et al. Drosophila Dscam is an axon guidance receptor exhibiting extraordinary molecular diversity. Cell. 2000:101:671-84.

4. Zhan XL, Clemens JC, Neves G, Hattori D, Flanagan JJ, Hummel T, et al. Analysis of Dscam diversity in regulating axon guidance in Drosophila mushroom bodies. Neuron. 2004;43:673-86.

5. Watson FL, Püttmann-Holgado R, Thomas F, Lamar DL, Hughes M, Kondo $M$, et al. Extensive diversity of Ig-superfamily proteins in the immune system of insects. Science. 2005;309:1874-8.

6. Chen BE, Kondo M, Garnier A, Watson FL, Püettmann-Holgado R, Lamar DR, et al. The molecular diversity of Dscam is functionally required for neuronal wiring specificity in drosophila. Cell. 2006;125:607-20.

7. Dong YM, Taylor HE, Dimopoulos G. AgDscam, a hypervariable immunoglobulin domain-containing receptor of the Anopheles gambiae innate immune system. PLoS Biol. 2006;4:1137-46.

8. Soba P, Zhu S, Emoto K, Younger S, Yang SJ, HH Y, et al. Drosophila sensory neurons require Dscam for dendritic self-avoidance and proper dendritic field organization. Neuron. 2007;54:403-16.

9. Wojtowicz WM, Wu W, Andre I, Qian B, Baker D, Zipursky SL. A vast repertoire of Dscam binding specificities arises from modular interactions of variable lg domains. Cell. 2007;130:1134-45.

10. Hattori D, Chen Y, Matthews BJ, Salwinski L, Sabatti C, Grueber WB, et al. Robust discrimination between self and non-self neurites requires thousands of Dscam1 isoforms. Nature. 2009;461:644-8.

11. Miura SK, Martins A, Zhang KX, Graveley BR, Zipursky SL. Probabilistic splicing of Dscam1 establishes identity at the level of single neurons. Cell. 2013:155:1166-77.

12. Sun W, You XT, Gogol-Döring A, He HH, Kise Y, Sohn M, et al. Ultra-deep profiling of alternatively spliced Drosophila Dscam isoforms by circularization-assisted multi-segment sequencing. EMBO J. 2013;32:2029-38.

13. He HH, Kise Y, Izadifar A, Urwyler O, Ayaz D, Parthasarthy A, et al. Cellintrinsic requirement of Dscam1 isoform diversity for axon collateral formation. Science. 2014;344:1182-6.

14. Schmucker D, Chen B. Dscam and DSCAM: complex genes in simple animals, complex animals yet simple genes. Genes Dev. 2009;23:147-56.

15. Zipursky SL, Sanes JR. Chemoaffinity revisited: dscams, protocadherins, and neural circuit assembly. Cell. 2010;143:343-53.

16. Lefebvre JL, Kostadinov D, Chen WV Maniatis T, Sanes JR. Protocadherins mediate dendritic self-avoidance in the mammalian nervous system. Nature. 2012:488:517-21.

17. Zipursky SL, Grueber WB. The molecular basis of self-avoidance. Annu Rev Neurosci. 2013;36:547-68.

18. Wu Q, Maniatis TA. Striking organization of a large family of human neural cadherin-like cell adhesion genes. Cell. 1999;97:779-90.

19. Tasic B, Nabholz CE, Baldwin KK, Kim Y, Rueckert EH, Ribich SA, et al. Promoter choice determines splice site selection in protocadherin alpha and -gamma pre-mRNA splicing. Mol Cell. 2002;10:21-33.

20. Wang XZ, Su H, Bradley A. Molecular mechanisms governing Pcdh-gamma gene expression: evidence for a multiple promoter and cis-alternative splicing model. Genes Dev. 2002;16:1890-905.
21. Chen WV, Nwakeze CL, Denny CA, O'Keeffe S, Rieger MA, Mountoufaris G, et al. Pcdhac2 is required for axonal tiling and assembly of serotonergic circuitries in mice. Science. 2017;356:406-11.

22. Mountoufaris G, Chen WV, Hirabayashi Y, O'Keeffe S, Chevee M, Nwakeze $\mathrm{CL}$, et al. Multicluster Pcdh diversity is required for mouse olfactory neural circuit assembly. Science. 2017;356:411-4.

23. Armitage SA, Freiburg RY, Kurtz J, Bravo IG. The evolution of Dscam genes across the arthropods. BMC Evol Biol. 2012;12:53-67.

24. Brites D, Brena C, Ebert D, Du Pasquier L. More than one way to produce protein diversity: duplication and limited alternative splicing of an adhesion molecule gene in basal arthropods. Evolution. 2013;67:2999-3011.

25. Yue Y, Meng YJ, Ma HR, Hou SQ, Cao GZ, Hong WL, et al. A large family of Dscam genes with tandemly arrayed $5^{\prime}$ cassettes in Chelicerata. Nat Commun. 2016;7:11252.

26. Lee MS, Soubrier J, Edgecombe GD. Rates of phenotypic and genomic evolution during the Cambrian explosion. Curr Biol. 2013;23:1889-95.

27. Leite DJ, McGregor AP. Arthropod evolution and development: recent insights from chelicerates and myriapods. Curr Opin Genet Dev. 2016; 39:93-100.

28. Kopelman NM, Lancet D, Yanai I. Alternative splicing and gene duplication are inversely correlated evolutionary mechanisms. Nat Genet. 2005;37:588-9.

29. Gulia-Nuss M, Nuss AB, Meyer JM, Sonenshine DE, Roe RM, Waterhouse RM, et al. Genomic insights into the Ixodes scapularis tick vector of Lyme disease. Nat Commun. 2016;7:10507.

30. Yamakawa K, Huot YK, Haendelt MA, Hubert R, Chen XN, Lyons GE, et al. DSCAM: a novel member of the immunoglobulin superfamily maps in a down syndrome region and is involved in the development of the nervous system. Hum Mol Genet. 1998:7:227-37.

31. Celotto AM, Graveley BR. Alternative splicing of the drosophila Dscam pre-mRNA is both temporally and spatially regulated. Genetics. 2001; 159:599-608

32. Neves G, Zucker J, Daly M, Chess A. Stochastic yet biased expression of multiple Dscam splice variants by individual cells. Nat Genet. 2004;36:240-6.

33. Meijers R, Puettmann-Holgado R, Skiniotis G, Liu JH, Walz T, Wang JH, et al. Structural basis of Dscam isoform specificity. Nature. 2007;449:487-91.

34. Schreiner D, Weiner JA. Combinatorial homophilic interaction between gamma-protocadherin multimers greatly expands the molecular diversity of cell adhesion. Proc Natl Acad Sci U S A. 2010;107:14893-8.

35. Goodman KM, Rubinstein R, Thu CA, Bahna F, Mannepalli S, Ahlsén G, et al. Structural basis of diverse homophilic recognition by clustered $\alpha$ - and $\beta$ protocadherins. Neuron. 2016;90:709-23.

36. Shapiro L, Love J, Colman DR. Adhesion molecules in the nervous system: structural insights into function and diversity. Annu Rev Neurosci. 2007;30:451-74.

37. Ravi V, WP Y, Pilla NE, Lian MM, Tay BH, Tohari S, et al. Cyclostomes lack clustered protocadherins. Mol Biol Evol. 2016;33:311-5.

38. Hulpiau P, van Roy F. New insights into the evolution of metazoan cadherins. Mol Biol Evol. 2011;28:647-57.

39. Guo Y, Xu Q, Canzio D, Shou J, Li JH, Gorkin DU, et al. CRISPR inversion of CTCF sites alters genome topology and enhancer/promoter function. Cell. 2015;162:900-10.

40. Hozumi N, Tonegawa S. Evidence for somatic rearrangement of immunoglobulin genes coding for variable and constant regions. Proc Natl Acad Sci. 1976;73:3628-32

41. Tonegawa S. Somatic generation of antibody diversity. Nature. 1983;302: 575-81.

42. Du Pasquier L, Zucchetti I, Santis RD. Immunoglobulin superfamily receptors in protochordates: before RAG time. Immunol Rev. 2004;198:233-48.

43. Kurtz J, Armitage SAO. Alternative adaptive immunity in invertebrates. Trends Immunol. 2006:27:493-6.

44. Sanggaard KW, Bechsgaard JS, Fang X, Duan J, Dyrlund TF, Gupta V, et al. Spider genomes provide insight into composition and evolution of venom and silk. Nat Commun. 2014:5:3765

45. Cao ZJ, Yu Y, YL W, Hao P, Di ZY, He YW, et al. The genome of Mesobuthus martensii reveals a unique adaptation model of arthropods. Nat Commun. 2013:4:2602.

46. Langmead B, Salzberg SL. Fast gapped-read alignment with bowtie 2. Nat Methods. 2012:9:357-9.

47. Mitchell A, Chang HY, Daugherty L, Fraser M, Hunter S, Lopez R, et al. The InterPro protein families database: the classification resource after 15 years. Nucleic Acids Res. 2015;43:D213-21. 
48. Sigrist CJ, de Castro E, Cerutti L, Cuche BA, Hulo N, Bridge A, et al. New and continuing developments at PROSITE. Nucleic Acids Res. 2013;41(Database issue):D344-7.

49. Larkin MA, Blackshields G, Brown NP, Chenna R, McGettigan PA, McWilliam $H$, et al. Clustal W and Clustal X version 2.0. Bioinformatics. 2007;23:2947-8.

50. Kumar S, Stecher G, Tamura K. MEGA7: molecular evolutionary genetics analysis version 7.0 for bigger datasets. Mol Biol Evol. 2016;33:1870-4.

51. Ganot P, Zoccola D, Tambutté E, Voolstra CR, Aranda M, Allemand D, et al. Structural molecular components of septate junctions in cnidarians point to the origin of epithelial junctions in eukaryotes. Mol Biol Evol. 2015;32:44-62.

52. Regier JC, Shultz JW, Zwick A, Hussey A, Ball B, Wetzer R, et al. Arthropod relationships revealed by phylogenomic analysis of nuclear protein-coding sequences. Nature. 2010;463:1079-83.

Submit your next manuscript to BioMed Central and we will help you at every step:

- We accept pre-submission inquiries

- Our selector tool helps you to find the most relevant journal

- We provide round the clock customer support

- Convenient online submission

- Thorough peer review

- Inclusion in PubMed and all major indexing services

- Maximum visibility for your research

Submit your manuscript at www.biomedcentral.com/submit
Biomed Central 